\title{
Fungal secondary metabolite dynamics in fungus-grazer interactions: novel insights and unanswered questions
}

\author{
Marko Rohlfs* \\ Johann-Friedrich-Blumenbach Institute of Zoology and Anthropology, Georg-August-University Göttingen, Göttingen, Germany
}

\section{Edited by:}

Ozgur Bayram, National University of Ireland Maynooth, Ireland

\section{Reviewed by:}

Jeffrey William Cary, United States

Department of Agriculture -

Agricultural Research Service, USA

Frank Kempken, Christian-Albrecht

Kiel University, Germany

\section{*Correspondence:}

Marko Rohlfs, Johann-Friedrich-

Blumenbach Institute of Zoology and Anthropology, Georg-August-University Göttingen, Berliner Strasse 28,

37073 Göttingen, Germany

e-mail:mrohlfs@gwdg.de
In response to fungivore grazing fungi are assumed to have evolved secondary metabolitebased defense mechanisms that harm and repel grazers, and hence provide a benefit to the metabolite producer. However, since research into the ecological meaning of highly diverse fungal secondary metabolites is still in its infancy, many central questions still remain. Which components of the enormous metabolite diversity of fungi act as direct chemical defense mechanisms against grazers? Is the proposed chemical defense of fungi induced by grazer attack? Which role do volatile compounds play in communicating noxiousness to grazers? What is the relative impact of grazers and that of interactions with competing microbes on the evolution of fungal secondary metabolism? Here, I briefly summarize and discuss the results of the very few studies that have tried to tackle some of these questions by (i) using secondary metabolite mutant fungi in controlled experiments with grazers, and by (ii) investigating fungal secondary metabolism as a flexible means to adapt to grazer-rich niches.

Keywords: Aspergillus, chemical defense regulation, fungivory, inducible resistance, secondary metabolites

\section{INTRODUCTION}

Fungal metabolism is an important source of an apparently endless diversity of organic compounds which are not obviously required for the producer's normal growth and metabolism - one reason why they are called secondary metabolites (SMs). This chemical diversity raises a pertinent yet hardly addressed basic research question in fungal biology: why are SMs produced in such variety, especially since the SM biosynthesis is encoded in clusters of genes whose transcriptional activation is embedded in a tightly regulated network comprising the activity of signaling pathways, epigenetic regulators, fungal hormones, and cellular secretion processes (Tsitsigiannis and Keller, 2007; Roze etal., 2011; Brakhage, 2013)?

Because many fungal SMs are toxic to other organisms, in particular animals, one line of arguments follows the idea that fungi produce specific SMs as direct defense compounds to achieve protection from grazers foraging for nutrient-rich food sources (e.g., Janzen, 1977; Martin, 1979; Camazine et al., 1983; Vining, 1990; Gloer, 1995; Spiteller, 2008). Putative direct defense compounds have emerged primarily due to pharmacological evidence for their deleterious effects on grazers. This evidence stems from studies that report relationships between fungivore mortality, decreased fecundity, and feeding on purified compounds mixed into artificial diets or on fungi expressing the proposed chemical defense trait constitutively (e.g., Wright et al., 1980; Paterson et al., 1987; Panigrahi, 1993; Gloer, 1995; Rohlfs and Obmann, 2009). Such studies provide at best weak correlative evidence as they do not evaluate whether these compounds increase fungal fitness under grazing pressure, which is the defining criterion of a chemical compounds-based defense.
In a series of recent studies (see below), the use of genetically modified fungi manipulated in the expression of candidate defense gene products has corroborated the relationship between SM regulatory mechanisms and resistance against grazers, yet manipulation of pathway-specific defense products have revealed conflicting results. Moreover, first studies have demonstrated complex chemical changes in fungi after fungivore attack, which could in part be related to changes in the capacity to resist grazing. The present review intends to provide a critical discussion of these recent findings and stimulate more research into the ecological causes and consequences of fungal chemical diversity.

\section{INVESTIGATING FUNGAL RESISTANCE WITH GENETICALLY MANIPULATED FUNGI}

Since more than a decade, functional genetic approaches have provided unparalleled insights into how fungi regulate their chemical diversity. Despite the availability of an increasing number of wellcontrolled mutant fungi, functional evaluations have largely been restricted to biochemical and medical aspects of fungal biology. Very few studies have used genetically modified fungi to conduct functional analyses of SM genes in the context of interactions with grazers.

\section{GLOBAL SM REGULATION}

Six of these studies focused on Aspergillus spp. laeA loss-offunction mutants in interactions with different soil arthropods, Collembola, (Rohlfs et al., 2007; Janssens et al., 2010; Staaden et al., 2011; Stötefeld et al., 2012) or facultative fungivorous Drosophila melanogaster larvae (Trienens et al., 2010; Caballero Ortiz et al., 2013). The methyltransferase-domain protein LaeA is essential for both appropriate developmental processes coupled with the biosynthesis of various SMs, including mycotoxins, such as 
sterigmatocystin (Bok and Keller, 2004; Sarikaya Bayram et al., 2010). Chemical deficient $\Delta$ laeA Aspergillus sp. mutants generally are less detrimental to grazer fitness than wild type fungi (reduced mortality, enhanced reproductive output) and, when given the choice, fungivores prefer grazing on the $\Delta$ laeA mutants. The latter appears to be mediated by a combined effect of differences in both the non-volatile SM and the volatile chemical profile (Staaden et al., 2011; Stötefeld et al., 2012). The LaeA-dependent capacity to harm insect grazers seems to be fungal species-specific though: while $\Delta$ laeA Aspergillus nidulans almost entirely lost the potential to kill fruit fly $D$. melanogaster larvae, $\Delta$ laeA $A$. flavus and $\triangle$ laeA $A$. fumigatus were still fatal to the insects, yet the onset of larval mortality was significantly delayed (Trienens et al., 2010). Importantly, compared to wild type, the lack of expression of laeA in all fungi tested increased their susceptibility to grazer damage (Rohlfs et al., 2007; Trienens et al., 2010; Stötefeld et al., 2012).

Loss of the capacity to kill fruit fly larvae was also evident for $A$. nidulans impaired in the expression of VeA (Trienens and Rohlfs, 2012), which indicates that an intact velvet (VelB-VeA-LaeA) complex (Bayram et al., 2008) is required for building resistance against grazers. Therefore, global regulatory mechanisms controlling both SM diversity and development have a distinct function in mediating protection from fungivore attack, and I would predict a similar role of these mechanisms in fungi beyond Aspergillus.

\section{PATHWAY-SPECIFIC SM REGULATION}

A review of the literature indicates that there are only five published studies that have used pathway-specific mutant strains to test for the influence of single SMs on the outcome of interactions with fungivores (Scheu and Simmerling, 2004; Staaden et al., 2010; Trienens and Rohlfs, 2012; Yin et al., 2012; Cary et al., 2014). As demonstrated by the use of knock-out mutants in feeding assays with Collembola, Folsomia candida and Protaphorura armata, and the nitidulid beetle Carpophilus freemani, polyketide synthase-driven formation of pigments dihydroxynaphthalene melanin in A. fumigatus conidia and asparasone A in sclerotia of $A$. flavus proved to impair or deter grazers, respectively, (Scheu and Simmerling, 2004; Cary et al., 2014). The latter is of particular interest because it is the first demonstration of a grazer-deterring compound whose biosynthesis is confined to fungal tissue that has a special function in ensuring survival under unfavorable conditions. However, how such pigments contribute to directly repelling grazers is unknown.

The polyketide sterigmatocystin is a characteristic mycotoxin of A. nidulans, which has insecticidal properties (Chinnici et al., 1983; Rohlfs and Obmann, 2009). In accordance with the proposed role of sterigmatocystin in mediating resistance against grazers, Collembola avoided feeding on a mutant $A$. nidulans strain over-expressing the bZIP transcription factor gene, $r s m A$, which results in a great increase of sterigmatocystin (Yin et al., 2012). RsmA activates the C6 transcription factor AflR, the ST pathway-specific regulatory factor required for transcriptional activation of ST biosynthetic genes. One would expect a benefit to grazers when exposed to A. nidulans deficient in the formation of sterigmatocystin. Interestingly, this hypothesis was not supported by Trienens and Rohlfs (2012). Compared with mortality in the presence of sterigmatocystin-producing wild type A. nidulans, Drosophila larvae did not demonstrate increased survival when confronted with 1-day old A. nidulans mutants $(\Delta a f l R, \Delta s t c J, \Delta s t c E, \Delta s t c U)$ incapable of producing sterigmatocystin. To our surprise, when the insects were exposed to initially 2-days old colonies, larvae suffered even higher mortality on substrate infested with the sterigmatocystin deficient mutant strains (Trienens and Rohlfs, 2012). A similar grazer response was observed when F. candida was offered a sterigmatocystin deficient $\triangle$ aflR $A$. nidulans mutant: relative to the wild type strains (Rohlfs et al., 2007), the Collembola suffered unusually high mortality and did not reproduce whatsoever (Albert, 2007). Thus, even though an artificial increase in sterigmatocystin biosynthesis enhances the capacity of $A$. nidulans to resist grazing, the loss of this compound does not reduce resistance as one would expect.

In conclusion, sterigmatocystin does not seem to be the major anti-grazer compound in wild type A. nidulans. In contrast, sterigmatocystin biosynthesis appears to hamper the ability of A. nidulans to develop even better protection against grazers. In search for an explanation of this phenomenon, one could argue that there are only a few key intermediates of the basic metabolic pathways that provide the starting points for the SM pathways. For example, acetyl-CoA is the precursor molecule for compound biosynthesis from the polyketide and the isoprenoid pathway. Possibly, artificial inactivation of the sterigmatocystin pathway leads to higher amounts of acetyl-CoA available for shunting into other pathways which produce more efficient anti-grazer compounds. If this turns out to be an adequate explanation for sterigmatocystin deficient $A$. nidulans being more detrimental to grazers than the wild type, fungi may suffer ecological costs of (high) SM diversity. And perhaps global, e.g., LaeA-dependent, regulatory mechanisms might prove to constrain fungi in activating the optimal combination of pathways for conquering grazers with a less diverse but more effective blend of SMs.

\section{RESPONSES OF FUNGI TO GRAZERS}

It has been well appreciated that fungal chemical phenotypes can vary with abiotic conditions such as light, water, temperature or the availability of nutrients (Schmidt-Heydt et al., 2008; Atoui et al., 2010; Schmidt-Heydt et al., 2010; Nielsen et al., 2011). Also, interactions with bacteria have been established as critical determinants of fungal SM composition (Brakhage and Schroeckh, 2011). Some recent analyses of the chemical responses to animal grazers provide first evidence of an inducible chemical compound-based defense response in fungi, which comprises the biosynthesis of so-called cryptic metabolites.

\section{EVIDENCE FROM Aspergillus nidulans}

Grazing by Collembola, F. candida, was found to induce an increase in the formation of sterigmatocystin, some meroterpenoids (mixed polyketide/terpenoid orign) and emericellamides (mixed polyketide/peptide origin) in A. nidulans (Döll et al., 2013). In choice experiments, Collembola preferred un-grazed colonies to grazed, probably due to changes in the volatiles released by damaged colonies (Staaden et al., 2011), and when forced to feed 


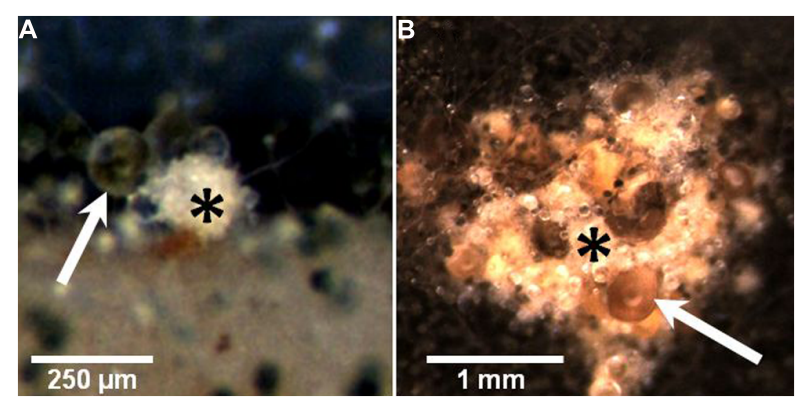

FIGURE 1 | Images depicting the localized formation of guttation droplets on the sexual fruiting bodies (cleistothecia) of $\boldsymbol{A}$. nidulans, which are possibly involved in mediating protection from grazing by Collembola (see Döll et al., 2013). (A) To the left of the initial stage (primodium) of a cleistothecium (asterisk) a strikingly large droplet (arrow) is formed in addition to some smaller ones. The whitish appearance of the primordium is due to layers of so-called Hülle cells which are assumed to nurse and protect the developing ascospores within the cleistothecium. It is not obvious whether the guttation droplets are produced by the Hülle cells or aerial hyphae surrounding the fruiting bodies. (B) A cluster of larger cleistothecia (asterisk), surrounded by a dark mat of conidia-producing tissue. The cluster is covered by voluminous droplets of light-brown color (arrow). Numerous smaller and apparently colorless droplets attached to single aerial hyphae are also visible.

on un-grazed or grazed mold the animals grew slower on previously attacked colonies. Moreover, grazer-challenged A. nidulans colonies intensified significantly the formation of sexual fruiting bodies (cleistothecia), which appeared to be the only fungal tissue that was not consumed after prolonged grazing (Döll et al., 2013). Possibly, the positive correlation of the appearance of cleistothecia and the intensive formation of guttation droplets on the sexual fruiting bodies (Figure 1) is a means of protecting this valuable tissue from grazers. For example, guttation droplets produced by Penicillium and Stachybotrys are known to contain high amounts of toxic SMs (Gareis and Gareis, 2007; Gareis and Gottschalk, 2014), yet, no report exists on the SM content in A. nidulans guttation droplets exists (and whether it changes under grazer pressure). Nonetheless, at least for A. nidulans, combined investment in SM formation and sexual development seems to be a strategy to maintain high fitness in grazer-rich niches (Figure 2).

These findings are supported by an experiment that examined the effect of larval Drosophila grazing on the expression of $A$. nidulans genes. In the presence of the insect larvae, $A$. nidulans exhibited shifts in the transcriptional activity of many genes, including those involved in signal transduction, hormonal signaling, and SM biosynthesis (Caballero Ortiz et al., 2013); interestingly, laeA ranked highest among those genes that were found up-regulated in response to the insects. Comparable with the results of the Collembola- $A$. nidulans experiment described above, grazed wild type colonies were found to kill Drosophila fly larvae more rapidly than unchallenged ones. An intriguing finding here was that, in a subsequent experiment, D. melanogaster larvae were able to use a $\triangle$ laeA mutant of otherwise fatal $A$. nidulans as the only available food source that promoted development into adult flies (Caballero Ortiz et al., 2013). That is LaeA-mediated activation of

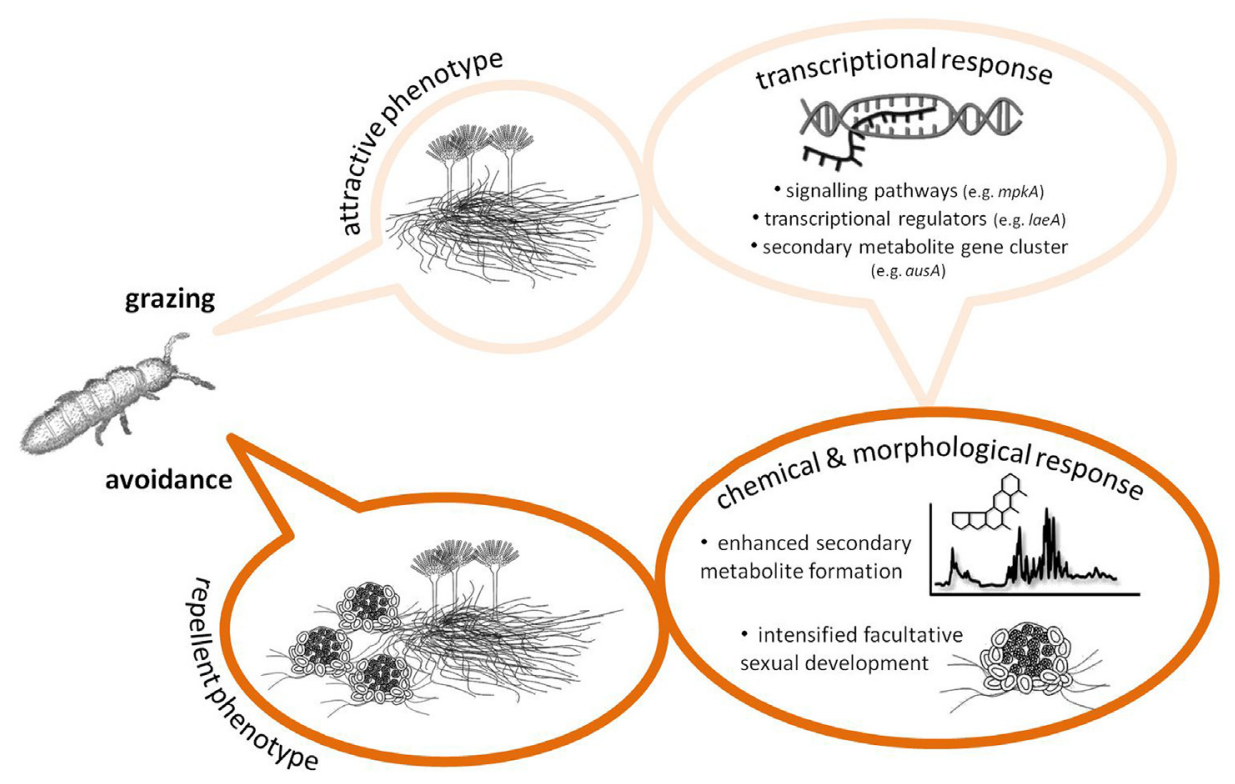

FIGURE 2 | Scheme summarizing the results of some recent studies demonstrating a grazer-induced defense response in the model fungus Aspergillus nidulans [see text and publications by Döll et al. (2013) and Caballero Ortiz et al. (2013) for details]. This scheme may serve as a modifiable blueprint for future studies providing evidence for or against an inducible chemical defense in fungi, add more specific information from other fungal systems, or contribute to general, system-independent properties of fungal chemical responses to grazers, e.g., hormone and pheromone signaling. Although not indicated in detail, it should also be specified how both putative defense compounds affect fungivore behavior and physiology, to be better able to determine the ecological consequences of fungal secondary metabolites (SM) biosynthesis and hence the selective forces that may have, at least in part, shaped fungal SM diversity. 
SM formation in response to grazing to a large extent prevents the fungus from being consumed and used as a suitable diet by these facultative fungivores.

Finally, terpenoid compounds that function as hormones in arthropods, the so-called juvenile hormones $(\mathrm{JH})$, have recently been found to be synthesized by $A$. nidulans, in particular under larval fruit fly grazing pressure (Nielsen et al., 2013). And when confronted with a JH over-expression mutant, fly larvae were significantly smaller. Possibly, the anti-grazer defense mechanisms of A. nidulans comprise the activity of insect hormone analogs that derange grazer endocrine processes.

Taken together, these findings illustrate the extent to which fungal morphological and chemical properties are affected by dynamic interactions with grazers and thereby reveal a number of new candidate defense pathways that may mediate direct resistance. Considering this chemical diversity of compounds we still lack convincing evidence for the identity of SMs used by fungi to repel and/or harm grazers. Even in this single A. nidulans system, it seems possible that more than one master defense compound exists, which are likely to interact with grazers via an array of perhaps equally diverse (patho-) physiological and behavioral mechanisms in the animals.

\section{EVIDENCE FROM OTHER FUNGI}

Using model fungal systems has many obvious advantages, yet there is a strong need to investigate the idea of inducible chemical compound-based resistance in other fungi. Outside the $A$. nidulans system, to the best of my knowledge, there is no good evidence of inducible chemical compound-based resistance in fungi. The study by Bleuler-Martínez et al. (2011) shows the induction of fruiting body lectins (carbohydrate-binding proteins) by nematode worms piercing hyphae and sucking in the cytoplasmatic content of Coprinopsis cinerea. In spite of this clear demonstration of an induced fungal response, it remains to be tested whether lectins provide an effective protection against the nematode or other grazers, since lectin toxicity has only been tested against nonfungivores (Bleuler-Martínez et al., 2011; Schubert et al., 2012; Žurga et al., 2014). Relevant to this discussion is the finding by Balogh etal. (2003), who did not find a positive influence of a lectin-deficient Arthrobotrys oligospora mutant on grazing by $F$. candida.

These very first studies illustrate the significance of grazing in determining the chemical profile of fungi but also the complexities and ambiguities involved in establishing a conceptually sound and direct connection between SM formation and fungal fitness. Therefore, separating functionally relevant from irrelevant chemical responses of fungi to grazing will be of utmost importance for the correct annotation of putative fungal defense traits that mediate protection from grazers. For example, genome-wide gene expression analyses should follow to reveal fungal responses to grazing the abovementioned studies may have overlooked.

\section{CONCLUSION}

We are only beginning to appreciate fungal chemical dynamics under varying ecological conditions, and we should investigate deeper the full repertoire, kinetics, and sites of secondary metabolite biosynthesis in response to grazers to be able to update and rearrange the lists of putative defense compounds (in $A$. nidulans and other fungi) on the basis of inducible reactions. Carefully conducted experiments with model grazers (e.g., Collembola, Drosophila larvae, nematodes, etc.) exposed to purified compounds will shed light on the mode of action in fungivores, e.g., whether fungal chemicals decrease or suppress the feeding response, act as acute toxins that interfere with intermediary metabolism or cellular functions, or cause chronic tissue and organ malfunctions which ultimately lead to impaired development and reduced fecundity. Finally, a large fraction of fungal SMs likely have evolved to allow effective control of and/or communication with other microbes in their environment, and we should thus think about the possibility that it is not the grazer itself that is directly affected by specific metabolites, but the endogenous microorganisms fungivores require for the control of their immune system, food breakdown, and detoxification. Thus, in order to fully appreciate the complete anti-grazer potential of SM biosynthesis in fungi, we need to combine their inducible dynamics with the influence of these compounds on grazer behavior, physiology, and multi-species interactions in their environment.

\section{ACKNOWLEDGMENT}

The author is funded by the German Research Foundation (Deutsche Forschungsgemeinschaft grant number: RO3523/3-2).

\section{REFERENCES}

Albert, M. (2007). Der Einfluss des Sekundärmetabolismus von Aspergillus nidulans auf Reproduktion, Überleben und Nahrungswahl pilzfressender Collembolen. Diploma thesis, Christian-Albrechts-University of Kiel, Germany.

Atoui, A., Kastner, C., Larey, C. M., Thokala, R., Etxebeste, O., Espeso, E. A., et al. (2010). Cross-talk between light and glucose regulation controls toxin production and morphogenesis in Aspergillus nidulans. Fungal Genet. Biol. 47, 962-972. doi: 10.1016/j.fgb.2010.08.007

Balogh, J., Tunlid, A., and Rosén, S. (2003). Deletion of a lectin gene does not affect the phenotype of the nematode-trapping fungus Arthrobotrys oligospora. Fungal Genet. Biol. 39, 128-135. doi: 10.1016/S1087-1845(03)0 0023-9

Bayram, Ö., Krappmann, S., Ni, M., Bok, J. W., Helmstaedt, K., Valerius, O., etal. (2008). VelB/VeA/LaeA complex coordinates light signal with fungal development and secondary metabolism. Science 320, 1504-1506. doi: 10.1126/science. 1155888

Bleuler-Martínez, S., Butschi, A., Garbani, M., Wälti, M. A., Wohlschlager, T., Potthoff, E., et al. (2011). A lectin-mediated resistance of higher fungi against predators and parasites. Mol. Ecol. 20, 3056-3070. doi: 10.1111/j.1365294X.2011.05093.x

Bok, J. W., and Keller, N. P. (2004). LaeA, a regulator of secondary metabolism in Aspergillus spp. Eukaryot. Cell 3, 527-535. doi: 10.1128/EC.3.2.527-53 5.2004

Brakhage, A. A. (2013). Regulation of fungal secondary metabolism. Nat. Rev. Microbiol. 11, 21-32. doi: 10.1038/nrmicro2916

Brakhage, A. A., and Schroeckh, V. (2011). Fungal secondary metabolites strategies to activate silent gene clusters. Fungal Genet. Biol. 48, 15-22. doi: 10.1016/i.fgb.2010.04.004

Caballero Ortiz, S., Trienens, M., and Rohlfs, M. (2013). Induced fungal resistance to insect grazing: reciprocal fitness consequences and fungal gene expression in the Drosophila-Aspergillus model system. PLoS ONE 8:e74951. doi: 10.1371/journal.pone.0074951

Camazine, S. C., Resch, J. F., Eisner, T., and Meinwald, J. (1983). Mushroom chemical defence. J. Chem. Ecol. 9, 1439-1447. doi: 10.1007/BF009 90749

Cary, J. W., Harris-Coward, P. Y., Ehrlich, K. C., Di Mavungu, J. D., Malysheva, S. V., De Saeger, S., et al. (2014). Functional characterization of a veA-dependent polyketide synthase gene in Aspergillus flavus necessary for the synthesis of 
asparasone, a sclerotium-specific pigment. Fungal Genet. Biol. 64, 25-35. doi: 10.1016/j.fgb.2014.01.001

Chinnici, J. P., Gunst, K., and Llewellyn, G. C. (1983). Effect of pretreatment of aflatoxin B1 post-treatment toxicity in Drosophila melanogaster (Diptera). J. Invertebr. Pathol. 41, 321-327. doi: 10.1016/0022-2011(83)90249-5

Döll, K., Chatterjee, S., Scheu, S., Karlovsky, P., and Rohlfs, M. (2013). Fungal metabolic plasticity and sexual development mediate induced resistance to arthropod fungivory. Proc. R. Soc. B Biol. Sci. 280, 20131219. doi: 10.1098/rspd.2013.1219

Gareis, M., and Gareis, E.-M. (2007). Guttation droplets of Penicillium nordicum and Penicillium verrucosum contain high concentrations of the mycotoxins ochratoxin A and B. Mycopathologia 163, 207-214. doi: 10.1007/s11046-0079003-1

Gareis, M., and Gottschalk, C. (2014). Stachybotrys spp. and the guttation phenomenon. Mycotoxin Res. 30, 151-159. doi: 10.1007/s12550-014-0193-3

Gloer, J. B. (1995). Antiisectan natural products from fungal sclerotia. Acc. Chem. Res. 28, 343-350. doi: 10.1021/ar00056a004

Janssens, T. K. S., Staaden, S., Scheu, S., Mariën, J., Ylstra, B., and Roelofs, D. (2010) Transcriptional responses of Folsomia candida upon exposure to Aspergillus nidulans secondary metabolites in single and mixed diets. Pedobiologia 54, 45-52. doi: 10.1016/j.pedobi.2010.09.002

Janzen, D. H. (1977). Why fruits rot, seeds mold and meat spoils. Am. Nat. 111, 691-713. doi: 10.1086/283200

Martin, M. M. (1979). Biochemical implications of insect mycophagy. Biol. Rev. 54, 1-21. doi: 10.1111/j.1469-185X.1979.tb00865.x

Nielsen, M. L., Nielsen, J. B., Rank, C., Klejnstrup, M. L., Holm, D. K., Brogaard, K. H., et al. (2011). A genome-wide polyketide synthase deletion library uncovers novel genetic links to polyketides and meroterpenoids in Aspergillus nidulans. FEMS Microbiol. Lett. 321, 157-166. doi: 10.1111/j.1574-6968.2011.0 2327.x

Nielsen, M. T., Klejnstrup, M., Rohlfs, M., Anyaogu, D. C., Nielsen, J. B., Gotfredsen, C. H., et al. (2013). Aspergillus nidulans synthesize insect juvenile hormones upon expression of a heterologous regulatory protein and in response to grazing by Drosophila melanogaster larvae. PLoS ONE 8:e73369. doi: 10.1371/journal.pone.0073369

Panigrahi, S. (1993). Bioassays of mycotoxins using terrestrial and aquatic, animal and plant species. Food Chem. Toxic. 31, 767-790. doi: 10.1016/02786915(93)90149-S

Paterson, R. R. M., Simmonds, M. S. J., and Blaney, W. M. (1987). Mycopesticidal effects of characterized extracts of Penicillium isolates and purified secondary metabolites (including mycotoxins) on Drosophila melanogaster and Spodoptora littoralis. J. Invertebr. Pathol. 50, 124-133. doi: 10.1016/0022-2011(87) 90112-1

Rohlfs, M., Albert, M., Keller, N. P., and Kempken, F. (2007). Secondary chemicals protect mould from fungivory. Biol. Lett. 3, 523-525. doi: 10.1098/rsbl.2007.0338

Rohlfs, M., and Obmann, B. (2009). Species-specific responses of dew fly larvae to mycotoxins. Mycotoxin Res. 25, 103-112. doi: 10.1007/s12550-0090015-1

Roze, L. V., Chanda, A., and Linz, J. E. (2011). Compartmentalization and molecular traffic in secondary metabolism: a new understanding of established cellular processes. Fungal Genet. Biol. 48, 35-48. doi: 10.1016/j.fgb.2010.05.006

Sarikaya Bayram, Ö., Bayram, Ö., Valerius, O., Park, H. S., Irniger, S., Gerke, J., et al. (2010). LaeA control of velvet family regulatory proteins for lightdependent development and fungal cell-type specificity. PLoS Genet. 6:e1001226. doi: 10.1371/journal.pgen.1001226

Scheu, S., and Simmerling, F. (2004). Growth and reproduction of fungal feeding collembola as affected by fungal species, melanin and mixed diets. Oecologia 139 347-353. doi: 10.1007/s00442-004-1513-7

Schmidt-Heydt, M., Magan, N., and Geisen, R. (2008). Stress induction of mycotoxin biosynthesis genes by abiotic factors. FEMS Microbiol. Lett. 284, 142-149. doi: 10.1111/j.1574-6968.2008.01182.x
Schmidt-Heydt, M., Rüfer, C. E., Abdel-Hadi, A., Magan, N., and Geisen, R. (2010) The production of aflatoxin B1 or G 1 by Aspergillus parasiticus at various combinations of temperature and water activity is related to the ratio of aflS to aflR expression. Mycotoxin Res. 26, 241-246. doi: 10.1007/s12550-010-0062-7

Schubert, M., Bleuler-Martinez, S., Butschi, A., Wälti, M. A., Egloff, P., Stutz, K., et al. (2012). Plasticity of the $\beta$-trefoil protein fold in the recognition and control of invertebrate predators and parasites by a fungal defence system. PLoS Pathog. 8:e1002706. doi: 10.1371/journal.ppat.1002706

Spiteller, P. (2008). Chemical defence of higher fungi. Chem. Eur. J. 14, 9100-9110. doi: 10.1002/chem.200800292

Staaden, S., Milcu, A., Rohlfs, M., and Scheu, S. (2010). Fungal toxins affect the fitness and stable isotope fractionation of Collembola. Soil Biol. Biochem. 42, 1766-1773. doi: 10.1016/j.soilbio.2010.06.014

Staaden, S., Milcu, A., Rohlfs, M., and Scheu, S. (2011). Olfactory cues associated with fungal grazing intensity and secondary metabolite pathway modulate Collembola foraging behaviour. Soil Biol. Biochem. 43, 1411-1416. doi: 10.1016/j.soilbio.2010.10.002

Stötefeld, L., Scheu, S., and Rohlfs, M. (2012). Fungal chemical defense alters density-dependent foraging behavior and success in a fungivorous soil arthropod. Ecol. Entomol. 37, 323-329. doi: 10.1111/j.1365-2311.2012.01373.x

Trienens, M., Keller, N. P., and Rohlfs, M. (2010). Fruit, flies, and filamentous fungi - experimental analysis of animal-microbe competition using Drosophila melanogaster and Aspergillus as a model system. Oikos 119, 1765-1775. doi: 10.1111/j.1600-0706.2010.18088.x

Trienens, M., and Rohlfs, M. (2012). Insect-fungus interference competition the potential role of global secondary metabolite regulation, pathway-specific mycotoxin expression and formation of oxylipins. Fungal Ecol. 5, 191-199. doi: 10.1016/j.funeco.2011.07.009

Tsitsigiannis, D. I., and Keller, N. P. (2007). Oxylipins as developmental and host-fungal communication signals. Trends Microbiol. 15, 109-118. doi: 10.1016/j.tim.2007.01.005

Vining, L. C. (1990). Function of secondary metabolites. Annu. Rev. Microbiol. 44, 395-427. doi: 10.1146/annurev.mi.44.100190.002143

Wright, V. F., Casas, E. D., and Harein, P. K. (1980). Evaluation of Penicillium mycotoxins for activity in stored-product Coleoptera. Environ. Entomol. 9, 217-221.

Yin, W.-B., Amaike, S., Wohlbach, D. J., Gasch, A. P., Chiang, Y.-M., Wang, C. C. C., et al. (2012). An Aspergillus nidulans bZIP response pathway hardwired for defensive secondary metabolism operates through aflR. Mol. Microbiol. 83, 1024-1034. doi: 10.1111/j.1365-2958.2012.07986.x

Žurga, S., Pohleven, J., Renko, M., Bleuler-Martinez, S., Sosnowski, P., Turk, D., et al. (2014). A novel $\beta$-trefoil lectin from the parasol mushroom (Macrolepiota procera) is nematotoxic. FEBS J. 281, 3489-506. doi: 10.1111/febs.12875

Conflict of Interest Statement: The author declares that the research was conducted in the absence of any commercial or financial relationships that could be construed as a potential conflict of interest.

Received: 07 November 2014; accepted: 22 December 2014; published online: 13 January 2015.

Citation: Rohlfs M (2015) Fungal secondary metabolite dynamics in fungus-grazer interactions: novel insights and unanswered questions. Front. Microbiol. 5:788. doi: 10.3389/fmicb.2014.00788

This article was submitted to Microbial Physiology and Metabolism, a section of the journal Frontiers in Microbiology.

Copyright (c) 2015 Rohlfs. This is an open-access article distributed under the terms of the Creative Commons Attribution License (CC BY). The use, distribution or reproduction in other forums is permitted, provided the original author(s) or licensor are credited and that the original publication in this journal is cited, in accordance with accepted academic practice. No use, distribution or reproduction is permitted which does not comply with these terms. 